\title{
Aiding the interpretation of forensic gait analysis: Development of a features of gait database
}

\author{
Ivan Birch ${ }^{\mathrm{a}, *}$, Claire Gwinnett ${ }^{\mathrm{b}}$, Jeremy Walker ${ }^{\mathrm{a}}$ \\ a Sheffield Teaching Hospitals NHS Foundation Trust, Jordanthorpe Health Centre, 1 Dyche Close, Sheffield, England S8 8DJ, United Kingdom \\ b Staffordshire University, College Road, Stoke-on-Trent, Staffordshire ST4 2DE, United Kingdom
}

\section{Introduction}

Forensic gait analysis is the use of gait and features of gait to assist in the process of identification, and it has now been presented in court for this purpose for 15 years [1]. Based on the experience of the authors and their colleagues, its use has increased during the last five years, with many police forces now considering forensic gait analysis as a forensic option, particularly with major crimes. Gait is the manner or style in which a locomotor activity, such as walking or running, is undertaken [2]. A person's gait is the result of a complex interaction of their anatomy, physiology, and in particular their neurology, pathology and injury, and represents a series of alterations, or compensations, to a basic pattern of movement necessitated by the individualities of the person. The resultant gait is then potentially further affected by additional intrinsic factors such as emotions and extrinsic factors such as footwear, terrain and the proximity of other individuals [3-6]. While gait is widely considered to be unique, its uniqueness is at a level that requires accurate and precise measurement of both kinetic and kinematic factors [7-9]. Current forensic gait analysis practice usually relies on the identification of features of gait from closed circuit television (CCTV) footage. This footage is often of poor quality in terms of resolution, lighting and frame rate, and is therefore limited in terms of the information it can provide [10]. It is also a two dimensional record of a three dimensional activity and incapable of yielding kinetic data. As a result forensic gait analysis as currently practiced is not capable of identifying a person. The features of gait that can be identified are class level features, that is to say features that occur in a proportion of the population, and therefore demonstrate compatibility rather than uniqueness. A fundamental skill of a forensic gait analyst is therefore an understanding of the prevalence of the features of gait identified in the population. To date this judgment has been based on past experience gained from sources such as past casework, clinical practice, text books, and published case studies and research papers [11-13]. Information gained from clinical practice requires careful consideration and use. If the practitioner is a specialist in musculoskeletal conditions, it may be expected that the majority of patients that seek their services have a musculoskeletal disorder or injury. As a result the prevalence of some features of gait,

\footnotetext{
* Corresponding author.

E-mail addresses: ivanbirch@btinternet.com (I. Birch), C.Gwinnett@staffs.ac.uk (C. Gwinnett), jeremy.walker@nhs.net (J. Walker).
}

based on such a sample of individuals, could be over-estimated. The data gained from these sources has in the past often been supplemented by unpublished ad-hoc surveys carried out by the forensic gait analyst. While such surveys can provide useful information they are particularly prone to skew in terms of demography caused by the location at which they occur. For example, if the data is collected at a location close to numerous healthcare facilities, it is possible that the individuals sampled will have a higher than usual prevalence of health related conditions. In addition to this, these ad hoc surveys are generally limited in size and scope, and although they provide some information regarding the prevalence of certain gait features, they are not able to represent the population in general. Clinical practice and ad-hoc surveys are a valuable source of knowledge regarding prevalence, but have to be used with caution, and should be supplemented wherever possible by reference to published texts and research papers. However, such texts and research papers can only provide an additional but limited source of information based on the specific purpose for which the publication was originally produced. This specific purpose, such as professional education, or a particular research question or subject group, may limit the transferability or currency of the information. The development of databases to hold information on forensic samples and observations has allowed a more robust interpretation of evidence and greater meaning of conclusions in court. Forensic databases have allowed numerical values to represent how common the feature of interest is in the environment and, for known provenance databases, to help identify a sample by providing a match between a suspect sample and a sample of known provenance. The production of these statistics, based on sound research and data collection, provides an objective interpretation of the evidence and enhances a forensic expert's opinion. Many forensic disciplines rely on the development of a database as the central point of reference for prevalence data and in view of the potential limitations of currently used sources of information relevant to forensic gait analysis, the development of a features of gait database is a fundamental step in the evolution of the discipline [14]. While the work described in this paper is a first step in this process, it should be noted that the database is still currently based on a subjective method, and further work needs to be undertaken before the database can legitimately be compared to those used by some other forensic disciplines.

The need for a features of gait database has been expressed by not only gait analysts but also other key individuals in the criminal justice system [15]. The need for underpinning data in order to reach sound 
conclusions for the court of law is particularly important when attempting to provide quantitative opinions utilising the Bayesian approach. The benefits of utilising the Bayesian approach in a range of forensic specialisms have been discussed extensively [16-20]. However, care must be taken when utilising this approach, to ensure transparency as to the origin and limitations of the data used. Failure to be transparent as to the data used to generate likelihood ratios led to conclusions based upon this approach being questioned in the Court of Appeal judgment in $\mathrm{R}$ v T [21].

In order for databases to be fit for use in the calculation of likelihood ratios and the interpretation of evidence in court, there are some key features that should be considered. Databases should contain an adequate number of samples so as to represent the population as appropriately as possible, be up-to-date (particularly if the samples are not static in their nature, for example fibre collections, where trends can change dramatically) and demonstrate scope, whether that be geographically and/or in the number of characteristics being collected/ analysed.

To ensure that databases and reference collections are a suitable basis on which to make inferences and aid in the interpretation of evidence, the Forensic Science Regulator's (FSR) Codes of Practice and Conduct 2016 outlines the minimum requirements for reference collections and databases [22]. These minimum requirements include the minimum quality standards for sample documentation, data accuracy and data entry. This paper reports the initial development and population of a gait database for use in the forensic context and where possible, outlines how this database currently complies with the Forensic Science Regulator's Codes of Practice and Conduct 2016. The limitations of this database with its current content are discussed along with suggestions of how this database will be developed further.

\section{Method}

\subsection{Database design}

Prior to method design and data collection, ethical approval was obtained through the research ethics approval process of Staffordshire University. In order to identify which features of gait should be included in the database, a Delphi strategy was employed to reach a consensus amongst a group of four expert practitioners in this field. While this number is low for a Delphi study, the area of practice concerned is highly specialized, and the number of practitioners known to be practicing in the field at the time in the United Kingdom was small (six) [23]. The Delphi strategy was originally developed as an interactive method of forecasting using a panel of experts to answer questions or give judgements in a series of developmental rounds [24]. After each round the outcomes are summarized and used to inform subsequent rounds, the experts progressively modifying their feedback as they feel appropriate, until a consensus is reached $[25,26]$. The four practitioners had considerable experience of forensic gait analysis, were all qualified at post graduate level, and trained in observational gait analysis. Each had a minimum of 20 years professional experience and 10 years involvement with forensic gait analysis. The participants were asked to list the features of gait they most commonly encountered and used during forensic gait analysis.

The outcomes of the first round of the exercise were summarized and reported back to the practitioners. The second round of the Delphi consolidated the findings and a consensus was reached. In the third round the practitioners were asked to review the list of features of gait identified in the context of the feasibility of observing and noting the features identified, in the time likely to be available during data collection, i.e. the time taken for a subject to walk towards, past and way from the observer. Consideration was given to a number of the available clinical observational gait analysis assessment tools [27-29]. It was concluded that the data collected using these tools was more detailed than it was feasible to collect in this instance, and relied on the ability to observe the subject for a protracted period of time. Consideration was also given to the likely validity of making the observations, particularly the accuracy with which estimations of magnitude could be made during data collection. It is important to note that the features of gait were therefore limited to those which could, with most certainty, be identified as being present or not present, with any estimations of magnitude being limited to ordinal categorical data, as shown on the data collection sheet. Demographic categories were modelled on a combination of the UK census categorisation, data from the Office for National Statistics [30], the views of the practitioners as to what might be useful in the forensic gait analysis context, and the feasibility of identifying the variables in the short time available.

Minor revisions were made to the list of features agreed in round two, and a consensus was reached in round four of the Delphi. A data collection sheet was produced, designed to facilitate the easy and rapid recording of the observed features of gait in the shortest possible time. The terminology used on the data collection sheet was intentionally kept simple, with the minimum use of technical terminology to ensure an unambiguous understanding of exactly what had been observed and to facilitate the later inclusion of additional data collection observers.

A pilot study was then undertaken of 20 randomly selected participants whose gait was observed, and as a result of the findings, minor amendments made to the data collection sheet to improve its usability under data collection conditions. The project was subsequently presented at national and international conferences attended by forensic gait analysts and their opinions sought as to the appropriateness of the features of gait identified during the Delphi process. The results have substantiated the selection of features made by the original four analysts. Table 1 shows the data collection sheet and features of gait observed.

\subsection{Data collection and storage}

Table 1

Data collection sheet showing the features of gait observed.

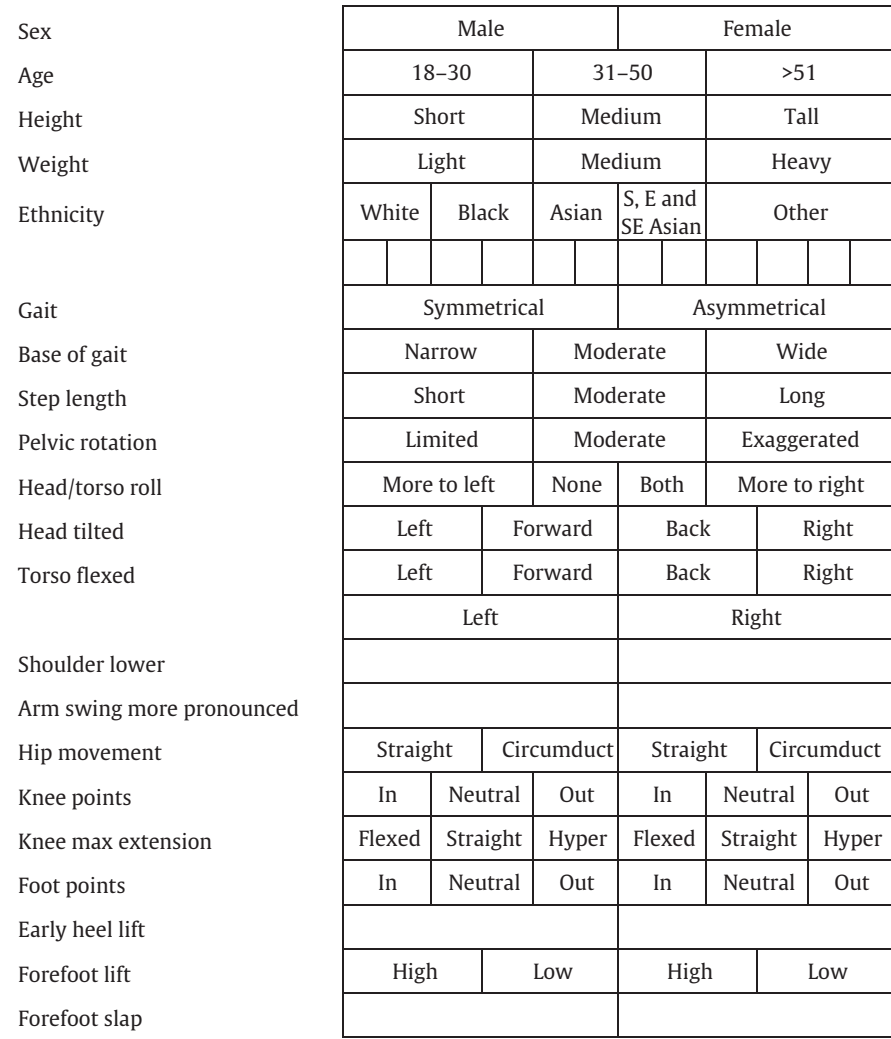


A single observer was utilized for the data collection. The observer had more than 35 years of experience in observational gait analysis, and was actively involved in forensic gait analysis practice, with more than 50 full forensic gait analysis reports having been produced and independently verified. This same individual is also responsible for the management of the database and will be referred to as the database manager from this point onwards. Members of the public were observed in a discreet manner, in public locations such as shopping centres and thoroughfares, at seven geographical locations across the country (Glasgow, Manchester, Birmingham, East London, West London, Brighton, Hastings). These initial locations were selected in order to maximise the opportunity for the widest selection of the UK population to be included in the database. Data collection was achieved by the observer positioning themselves in a suitable unobtrusive location, with a clear line of sight in at least two opposing directions, and a steady stream of pedestrians. Pedestrians were selected randomly for inclusion. This was achieved by selecting the fifth pedestrian to enter the data collection area after the completion of the previous data collection. Pedestrians were not selected for inclusion if they were:

- carrying a bag or bags likely to affect gait

- pushing a buggy or pushchair

- holding the hand of another person

- having their speed or direction of walking affected by an accompanying person

- walking with the aid of a stick or frame

Pedestrians were also not selected for inclusion, at the discretion of the observer, if they exhibited factors that were considered to be substantially altering their gait. Once selected for inclusion, observations were made working from the top of the data collection sheet progressively downwards. Observations were made as the subject walked towards, past and away from the observer. If a full set of data could not be collected from a subject, or the data collection sheet was not completed to the satisfaction of the observer, the data set was rejected. Approximately $7 \%$ of the attempted data sets were rejected due to being incomplete. In the majority of cases a minimum of 100 data sets were collected at each location, although fewer sets were collected opportunistically on some occasions.

\subsection{Documentation of the database}

As described in Section 20.19.4.5 in the Forensic Science Regulator's Code of Practice and Conduct [22], the following information has been outlined for the features of gait database;

\section{The purpose of the database,}

2. Where the database is currently located,

3. The current scope and content of the database,

4. The origin of the data collected for the database,

5. Any known significant limitations or restrictions of the data,

6. The person responsible for the management of the database,

7. The authorisation and competence requirements of practitioners contributing to the database,

8. The arrangements and format for data collection and entry,

9. The arrangements and format for data storage,

10. The quality assurance requirements, including those for data integrity, transfer, inconsistency and error checking,

11. All relevant ethical requirements covering the data content, retention, accessibility or use.

Where the above information has not been provided previously in this paper, it has been included in the results and discussion sections below.

\section{Results}

To date, data collection has taken place at seven locations around the UK, yielding 1007 data sets, each containing 28 pieces of information about the subject.

Following each data collection session, the data was transferred onto a Microsoft Access database, using a custom designed data entry form with all options for each piece of data being provided by dropdown menus. This task was not undertaken by the observer, but by an honours graduate who had no role in the development of the database other than data entry and checking. During data entry the integrity of each data set was scrutinised and any data sets identified as having missing or ambiguous data were rejected. During this process approximately $3 \%$ of the data sets submitted for entry into the database were rejected. Once the data from that collection session had been entered it was checked for omissions, inconsistencies and data entry errors by the database manager. Table 2 shows the demographic content of the database to date, based on the estimations of demographic variables by the observer. The database is currently housed on a protected computer, with password protection, at the database manager's office address.

\section{Discussion}

The purpose of building this database is to collect and store prevalence data on a range of gait features in the general population in order to aid the interpretation of gait evidence in court and increase the objectivity of any conclusions drawn. The Microsoft Access database constructed provides an easily interrogatable source of information on the prevalence of the features of gait included. Each feature of gait can be interrogated using a dropdown menu of options that heads each of the variable columns. For example the dropdown menu that heads the data regarding the roll of the head and torso lists the options of 'select all', 'blanks', 'more to the left', 'none', 'both' and 'more to the right'. One or more of the options can be selected, resulting in only the subjects who exhibited the selected options then being shown. The 'blanks' option is included to allow screening of the data sets following data entry, and to ensure that all data sets are complete. Using this system, the data can be interrogated one feature of gait at a time, or by a combination of features, a particular strength of the database. One of the significant weaknesses of ad-hoc surveys that collect data on a single feature of gait, is their inability to be able to discriminate between dependent and independent features. Even if the data from a series of single feature ad-hoc surveys is drawn upon, the interaction of dependent variables cannot be accommodated in the conclusions drawn from the data due to the fact that different samples have been observed. As a result, dependent variables are counted as independent variables, increasing the apparent significance of the combination of features

Table 2

Demographic content of the database to date, based on the estimations of demographic variables by the observer.

\begin{tabular}{|c|c|c|c|c|c|c|}
\hline \multirow[t]{2}{*}{ Demographic variable } & \multicolumn{2}{|c|}{$\begin{array}{c}\text { Number in } \\
\text { sample to date }\end{array}$} & \multicolumn{2}{|c|}{$\begin{array}{l}\text { Percentage of } \\
\text { sample }\end{array}$} & \multicolumn{2}{|c|}{$\begin{array}{l}\text { Percentage of } \\
\text { UK population } \\
\text { (based on } 2011 \\
\quad \text { census) }\end{array}$} \\
\hline & Male & Female & Male & Female & Male & Female \\
\hline Sex & 599 & 408 & 59.5 & 40.5 & 49 & 51 \\
\hline Age group 18-30 & 290 & 222 & 56.6 & 43.4 & 50.1 & 49.9 \\
\hline Age group 31-50 & 233 & 150 & 60.8 & 39.2 & 49.6 & 50.4 \\
\hline Age group $>51$ & 76 & 36 & 67.9 & 32.1 & 47.3 & 52.7 \\
\hline White & \multicolumn{2}{|c|}{783} & \multicolumn{2}{|c|}{$\frac{1}{77.8}$} & \multicolumn{2}{|c|}{87.2} \\
\hline Black & \multicolumn{2}{|c|}{87} & \multicolumn{2}{|c|}{8.7} & \multicolumn{2}{|c|}{3} \\
\hline Asian & \multicolumn{2}{|c|}{109} & \multicolumn{2}{|c|}{10.8} & \multicolumn{2}{|c|}{4.9} \\
\hline South, East and Southeast Asian & \multicolumn{2}{|c|}{19} & \multicolumn{2}{|c|}{1.9} & \multicolumn{2}{|c|}{2} \\
\hline Other & \multicolumn{2}{|c|}{8} & \multicolumn{2}{|c|}{0.8} & \multicolumn{2}{|c|}{2.9} \\
\hline
\end{tabular}


seen. By using the sequential selection of the options from the dropdown menus for the features of gait observed from CCTV footage, the database accommodates the relationship between dependent variables. For example, utilising the current data held in the database, the prevalence of the right knee pointing inwards towards the midline of the body when flexed during the swing phase of gait is shown to be approximately 1 in 14 (71 in 1007), and that of the right foot pointing inwards when weight bearing during the stance phase of gait to be approximately 1 in 17 (60 in 1007). If these two pieces of information are treated as completely independent, the prevalence of the two occurring together would be approximately 1 in 238 . However, the database shows the prevalence to be approximately 1 in 27 (37 in 1007), suggesting some level of dependency between the variables. The relationship between the occurrences of particular features of gait is an area that warrants further study, which will be enabled by the development of the database.

The database currently has a number of limitations. First and foremost, the database is predicated on subjective observations, made by one experienced observer. This method was selected in order to match as closely as possible the method currently used in forensic gait analysis, which is also based on subjective observations of features of gait. The use of a single observer for data collection ensured consistency in the data collection process, an important factor in this stage of the development of the database. However, the method used differs from that used in forensic gait analysis in that during forensic gait analysis, the footage can be played multiple times, providing a greater opportunity for the observation of features of gait than could be achieved using the real-time observation strategy adopted for data collection.

The robustness of a database is dependent upon the number of samples it contains, the ability of those samples to represent the population and the quality of the information that can be derived from the database [31]. While the current total of 1007 data sets represents the largest forensic gait analysis database known, it is a small sample, and the long term use as a resource will depend on its continued expansion. This expansion will ensure that data do not become out-ofdate and is proportionately representative of the various subsections of the UK population. Protocols must also be put in place as to the processes required to ensure the ongoing validity of the data and effectiveness of the database as outlined in the FSR's Codes section 20.19.4.5q [22].

In terms of the demographic distribution of the data collected to date, in comparison with the data yielded by the 2011 UK census, the white and 'other' populations are underrepresented in the database, while the black and Asian populations are overrepresented. The database sample is currently small in terms of the UK population, and such discrepancies are therefore perhaps to be expected as the sample was selected randomly in each geographical location. With the exception of 'other' and the South, East and Southeast Asian population which are reversed, the ranking of the frequencies of each demographic group matches that of the 2011 census data. It is also recognised that the demographic data is based on judgements made by the observer, and is therefore prone to a degree of error, as would such judgements made during analysis from CCTV footage. The current discrepancies will be monitored as the database grows.

A further limitation of the database is the number and range of features of gait included. As has been described, the number of features which could be recorded using the selected method, was limited by the nature and time constraints of real-time observation and data recording. As a result the database will not yield information regarding all the features of gait likely to be used in forensic gait analysis. The database will provide information regarding some of the most commonly used features; the method offering an appropriate compromise between collecting data for a more extensive range of features and the inherent ethical issues of videoing subjects without consent.

\section{Future development of the database}

Data collection will continue, but is currently limited by the use of a single experienced observer, which helps to ensure consistency and validity of the data collected. The use of this observer is believed to represent the most robust method of data collection at this stage in the development of the database. Further data will of course be collected, with the intention being for a limited number of additional observers, who are also deemed experts in forensic gait analysis, with the same or equivalent qualifications as the current observer, to be trained to increase the speed at which the database expands. Although it is tempting to plan for large numbers of contributors, the total number of observers will be kept to a minimum to assure the validity of the data collected. Once suitable candidates have been recruited a training event will take place prior to the collection of any data and the use of a competency test for additional contributors will be considered.

The development of this database is predicated on the notion of improving the validity of conclusions reached by forensic gait analysis, and this goal can only be achieved if the database is widely used. To this end, the database will ultimately be made accessible to all practitioners, upon request, who have undertaken appropriate training in its use, but with restricted access to prevent unauthorised viewing and contribution. It is hoped that in due course the database will be a password protected online resource for registered users, held centrally, but accessible from any suitably equipped piece of equipment.

As the database expands, with the intention of adding additional contributors/users and for the data to be used in casework, there will be a need for the development of further protocols. To ensure compliance of the features of gait database with the FSR's Codes of Practice and Conduct, the following protocols will be developed so as to allow further data entry and use. Where possible, direct reference to the requirements as stated in the FSR's Codes have been provided in brackets:

1. The process for making updates and amendments, and maintaining audit trails (20.19.4.5.k)

2. The protocols for access to the database and its interrogation and use (20.19.4.5.1)

3. The confidentiality and security requirements of accessing the database (20.19.4.5.n)

4. The format and content of results and reports from interrogation of the database, including the provision of any caveats relating to any limitations with the results provided (20.19.4.5.0)

5. The arrangements for review of relevance, use and effectiveness of the data (20.19.4.5.q)

\section{Conclusion}

In order to develop further, forensic gait analysis requires a robust database of the prevalence of features of gait. This study has attempted for the first time, to generate a database holding useable data on features of gait observed in the population in order to improve the interpretations of gait evidence in court. This database has been designed in accordance with the Forensic Science Regulator's Codes of Practice and Conduct and will continue to comply with these codes as it develops. Although currently small in size, the database can still assist the gait analyst in providing a more objective and meaningful interpretation of gait evidence as long as the data is used cautiously and transparently.

\section{Acknowledgements}

The authors would like to thank Sheffield Teaching Hospitals NHS Foundation Trust for funding the development of the features of gait database and the collection of data. 


\section{References}

[1] A. Buncombe, Gang Leader Is Unmasked by his Bandy-Legged Gait, The Independent, London, Jul, 13 2000, p. 115.

[2] D. Levine, J. Richards, M.W. Whittle, Whittle's Gait Analysis, Elsevier Health Sciences, 2012

[3] I. Birch, T. Birch, D. Bray, The identification of emotions from gait, Sci. Justice (2016) (in press, Available online 13 May 2016, Corrected Proof).

[4] W. Vernon, A. Parry, M. Potter, A theory of shoe wear pattern influence incorporating a new paradigm for the podiatric medical profession, J. Am. Podiatr. Med. Assoc. 94 (2004) 261-268.

[5] J.C. Menant, J.R. Steele, H.B. Menz, B.J. Munro, S.R. Lord, Effects of walking surfaces and footwear on temporo-spatial gait parameters in young and older people, Gait Posture 29 (2009) 392-397.

[6] S.B. Thies, J.K. Richardson, J.A. Ashton-Miller, Effects of surface irregularity and lighting on step variability during gait: a study in healthy young and older women, Gait Posture 22 (2005) 26-31.

[7] V.B. Semwal, M. Raj, G.C. Nandi, Biometric gait identification based on a multilaye perceptron, Robot. Auton. Syst. 65 (2015) 65-75.

[8] C. Yam, M.S. Nixon, J.N. Carter, Automated person recognition by walking and running via model-based approaches, Pattern Recogn. 37 (2004) 1057-1072.

[9] D. Kastaniotis, I. Theodorakopoulos, C. Theoharatos, G. Economou, S. Fotopoulos, A framework for gait-based recognition using Kinect, Pattern Recogn. Lett. 68 (2015) 327-335

[10] I. Birch, W. Vernon, G. Burrow, J. Walker, The effect of frame rate on the ability of experienced gait analysts to identify characteristics of gait from closed circuit television footage, Sci. Justice 54 (2014) 159-163.

[11] J.A. DiMaggio, W. Vernon, Forensic Podiatry: Principles and Methods, Humana, New York London, 2011

[12] T. Öberg, A. Karsznia, K. Öberg, Basic gait parameters: reference data for normal subjects, 10-79 years of age, J. Rehabil. Res. Dev. 30 (1993) 210.

[13] T.M. Owings, M.D. Grabiner, Variability of step kinematics in young and older adults, Gait Posture 20 (2004) 26-29.

[14] C. Champod, I.W. Evett, G. Jackson, Establishing the most appropriate databases for addressing source level propositions, Sci. Justice 44 (2004) 153-164.
[15] M. Wall, Paper Presented to Forensic Science Regulator's Annual Conference, Birmingham, 3rd March 2016, 2016.

[16] A. Biedermann, F. Taroni, S. Bozza, Implementing statistical learning methods through Bayesian networks. Part 1: a guide to Bayesian parameter estimation using forensic science data, Forensic Sci. Int. 193 (2009) 63-71.

[17] I.W. Evett, G. Jackson, J. Lambert, More on the hierarchy of propositions: exploring the distinction between explanations and propositions, Sci. Justice 40 (2000) 3-10.

[18] J. Skerrett, C. Neumann, I. Mateos-Garcia, A Bayesian approach for interpreting shoemark evidence in forensic casework: accounting for wear features, Forensic Sci. Int. 210 (2011) 26-30.

[19] I.W. Evett, J.A. Lambert, J.S. Buckleton, A Bayesian approach to interpreting footwear marks in forensic casework, Sci. Justice 38 (1998) 241-247.

[20] R. Cook, I.W. Evett, G. Jackson, P. Jones, J. Lambert, A hierarchy of propositions: deciding which level to address in casework, Sci. Justice 38 (1998) 231-239.

[21] R.v. T, EWCA Crim 2439, (2010).

[22] F.S. Regulator, Codes of Practice and Conduct: For Forensic Science Providers and Practitioners in the Criminal Justice System, 2016.

[23] W. Vernon, The Delphi technique: a review, Int. J. Ther. Rehabil. 16 (2009) 69-76

[24] K.C. Green, J.S. Armstrong, A. Graefe, Methods to elicit forecasts from groups: Delphi and prediction markets compared, Foresight: The International Journal of Applied Forecasting 2007 (2007) 17-20.

[25] H.A. Linstone, M. Turoff, The Delphi Method: Techniques and Applications, AddisonWesley Reading, MA, 1975.

[26] C. Okoli, S.D. Pawlowski, The Delphi method as a research tool: an example, design considerations and applications, Inf. Manag. 42 (2004) 15-29.

[27] H.S. Read, M.E. Hazlewood, S.J. Hillman, R.J. Prescott, J.E. Robb, Edinburgh visual gait score for use in cerebral palsy, J. Pediatr. Orthop. 23 (2003) 296-301.

[28] B. Toro, C.J. Nester, P.C. Farren, The development and validity of the Salford Gait Tool: an observation-based clinical gait assessment tool, Arch. Phys. Med. Rehabil. 88 (2007) 321-327.

[29] J. Perry, Gait Analysis, Los Amigos Research \& Education Center, Downey, California, 2002

[30] O.f.N. Statistics, 2011 UK Census, (2011)

[31] K. Pye, S.J. Blott, Development of a searchable major and trace element database for use in forensic soil comparisons, Sci. Justice 49 (2009) 170-181. 